\title{
EMPIRICAL BAYESIAN BEFORE-AND-AFTER ANALYSIS OF TREATMENTS: SIMULATED TRAFFIC CONFLICT APPROACH
}

\author{
Usama Elrawy Shahdah ${ }^{1}$ \\ ${ }^{1}$ Public Works Engineering Department, Faculty of Engineering, Mansoura University, Mansoura, Egypt
}

Received 28 August 2019; accepted 12 November 2019

\begin{abstract}
An integrated empirical Bayesian (EB) model based on crash-conflict safety performance function (SPF) as a prior is presented for estimating treatment effects at treated sites. This model has the advantage of accounting for changes in before and after factors not associated with the treatment, such as changes in volume and other site-specific attributes. The model also has the potential to reduce the need for large reference sites to develop the crash prediction safety performance function (SPF) in the EB formulation. The integration of traffic conflicts in EB before-and-after analysis can be achieved in two steps: 1) Develop a SPF to relate crashes to simulated conflicts for untreated (reference) sites, and 2) Simulate traffic at the treated sites with and without the treatment. The former serves as the prior in the EB formulation, while the latter accounts for changes in conflicts resulting from the treatment being considered and serves as the EB data likelihood. To evaluate the proposed crash-conflict model, an EB before and after analysis was performed for a sample of treated signalized intersections from Toronto, Canada. The treatment being considered at these intersections is changing the priority of left turn signals from permissive to protected-permissive control. Treatment effect is obtained in terms of Crash Modification Factors (CMFs) for rear-end and left-turn opposing (LTOPP) simulated traffic conflicts. CMFs obtained from the proposed crash-conflict model were compared with estimates of CMF from a convention
\end{abstract}

Keywords: empirical Bayesian, before-and-after analysis, traffic conflicts, surrogate safety measures, signalized intersections.

\section{Introduction}

Observational crash-based models have been traditionally used to estimate treatment effects in road safety studies. These models require the specification of a safety performance function (SPF) relating historical crash occurrence to selected sitespecific confounding factors, such as, traffic and road geometric attributes. The higher the number of confounding factors, the greater the number of sites that need to be considered to establish the SPF. The level of uncertainty associated with SPFs is represented by the underlying model variance for the expected number of crashes. It is worth noting that, SPF alone cannot not be used to estimate treatment effect because it cannot account for regression-to-the-mean (RTM) bias inherent in the observed crash data.

The most commonly accepted "observational" approach for estimating treatment effects and accounting for RTM bias is the empirical

${ }^{1}$ Corresponding author: usama.shahdah@mans.edu.eg 
Bayesian (EB) before-and-after approach (Hauer, 1997; Persaud and Lyon, 2007; etc.). RTM bias is accounted for in the EB approach by expressing the EB expected number of crashes at a given site as a weighted function of the:

1. Observed crashes at a given site; and

2. Predicted crashes for the site from the SPF.

In order to get reliable results for EB applications, a large sample of reference sites is needed (mostly untreated) from which to develop the underlying SPF, which is considered costly and impractical (Lan, 2010). As noted above, the level of uncertainty associated with the SPF prediction at a given site is represented by the underlying model variance and the expected number of crashes at that site. In the EB model, this uncertainty (or crash variance) is accounted for formally in the weight factor used to reflect confidence in the ability of the SPF expression to predict crashes at a given site. If the model is unreliable then more observational data is needed (i.e., more reference sites) to establish this SPF.

In the SPF function, the average annual daily traffic (AADT) is usually applied to reflect exposure and often this volume is the only independent variable introduced in the model. For urban areas AADT at intersections is not normally observed annually from counting stations, but is interpolated based on vehicles counts that are manually reported over shorter periods of time (usually 8 hours) and, this data collection is carried out every 2 to 6 years based on the importance of the intersection. In Toronto for example, 8-hour volumes are collected every 2 to 3 years at major intersections, and expansion factors are used to convert these hourly volumes to AADT (Tsoi, 2004). In addition, AADT is changing from year to year, which renders challenging our ability to account for annual volume changes in the resultant prediction models.

Recently, simulated vehicle interactions and traffic conflicts are used in traffic safety analysis, as surrogate safety measures instead of historical crash data. A conflict is observed when two or more vehicles approach each other, such that collision can take place if the vehicles trajectories unchanged (Amundsen and Hyden, 1977). The use of traffic conflicts in traffic safety analysis is based on that traffic conflicts can address some of the statistical issues associated with the rare random nature of crashes and reporting problems, especially for low severity crashes (Shahdah, 2014; Shahdah et al., 2014). In addition, the transportation failure mechanism, using traffic conflicts, is considered from a broader causal perspective than from observational analysis (Shahdah et al., 2014; Brown, 1994; Sayed et al., 1994; Van der Horst, 1990).

Microscopic traffic simulation platforms (e.g. VISSIM (PTV, 2012), SUMO (Lopez, 2018), AIMSUN (TSS, 2019) and PARAMICS (Quadstone, 2019)) can be used in the estimation of vehicle interactions and hence traffic conflicts (Gettman and Head, 2003; Cunto and Saccomanno, 2008; Shahdah et al., 2014; Shahdah et al., 2015; etc.)

The study introduced in this paper suggests that simulated traffic conflicts can be used in place of traffic volumes to enhance the performance of EB before-and-after analysis for estimating treatment effects. The integration of traffic conflicts in the EB model also promises to resolve the problem of a large sample size required for reference sites in establishing the SPF. 
The integration of traffic conflicts into the EB before-and-after analysis can be achieved in two steps:

1. Develop a SPF to relate crashes to simulated conflicts for untreated (reference) sites. This serves as the prior in the EB formulation;

2. Simulate the treated sites with and without treatment. This is used to account for changes in conflicts resulting from treatment only and serves as the data likelihood in the EB formulation.

To evaluate the proposed method, a before and after EB analysis was performed for a sample of treated signalized intersections from Toronto, Canada. The treatment considered at these intersections is to change priority of left turn signals from permissive to protected-permissive. Treatment effects are expressed in terms of Crash Modification Factors (CMFs). In this study, CMFs are established separately for rear-end (RE) and left-turn opposing (LTOPP) crashes. CMFs obtained from the proposed crash-conflict model are compared with estimates of CMF obtained from a conventional EB beforeand-after analysis for the same treatments at the same intersections used in this research paper.

\section{Traffic Conflict-Based EB Before-and- After Procedure}

In EB before-and-after analysis, CMF can be

estimated in terms of the ratio $(\theta)$ between the observed crashes in the after period $(\lambda)$ with treatment, and the expected number of crashes in the after period had the treatment not been implemented $(\pi)$ (Hauer, 1997), such that: $\theta=\frac{\lambda / \pi}{1+\left(\operatorname{Var}(\pi) / \pi^{2}\right)}$

With a variance of:

$\operatorname{VAR}(\theta)=\frac{\theta^{2}\left(\frac{\lambda}{\lambda^{2}}+\frac{\pi}{\pi^{2}}\right)}{\left(1+\frac{\operatorname{var}(\pi)}{\pi^{2}}\right)^{2}}$

where,

$\theta=$ crash modification factor,

$\lambda=$ the observed crashes in the after

period, and

$\pi=$ the expected crashes in the after

period had the treatment not been

implemented.

If the value of CMF $(\theta)$ is less than one, the treatment is considered effective in reducing crashes, and vice versa.

Before estimating $\pi$, the expected crashes in the before period is obtained from the EB expression. The expected number of crashes is expressed simply as:

$$
m_{i}=w_{i} \times \mathrm{E}\left(\mathrm{k}_{i}\right)+\left(1-w_{i}\right)\left(K_{i}\right)
$$

where,

$m_{i}=$ Expected number of crashes at site i, $E\left(\mathrm{k}_{i}\right)=$ Expected crashes at site $\mathrm{i}$

estimated from SPFs,

$K_{i}=$ Observed crash counts at site i, and $w_{i}=$ Weight factor.

The weight "w" is expressed as: 


$$
w_{i}=\frac{1}{\left(1+\varphi \times E\left(k_{i}\right)\right)}
$$

The term ö in Equation (4) is referred to as the over-dispersion parameter for the underlying crash frequency distribution. The weight factor value ranges between 0 and 1 . The lower the variance of prediction, the closer the weight factor is to a value of 1.0, and the greater the emphasis on the expected number of crashes from SPF function, and vice versa.

A factor (Cr) is then applied to $m_{i}$ (Eq. 3) to account for different conditions between the before and the after periods. In the conventional EB approach, these changes are usually traffic volumes and other confounding factors. The factor $\mathrm{C}_{\mathrm{r}}$ can be expressed as the ratio of the expected number of crashes in the after period and the expected number of crashes in the before period, as obtained from the SPF model.

The expected number of crashes in the after period had the treatment not been implemented $(\pi)$ can simply be estimated by multiplying the expected number of crashes in the before period $(\mathrm{m})$ by the correction factor (Cr).

This paper takes the position that simulated traffic conflicts can be used in place of traffic volumes to enhance the performance of EB before-and-after analysis for estimating treatment effects. The integration of traffic conflicts in the EB model also promises to resolve the problem of a large sample size required for reference sites in establishing the SPF in the conventional EB before-andafter analysis. The suggested SPF model used, in this paper, to estimate the expected crashes, is expressed in terms of the expected simulated conflicts, such that:

$$
L N\left(C_{i}\right)=L N(\alpha)+\beta L N\left(C F_{i}\right)
$$

where,

$C_{i}=$ the expected crashes at site $\mathrm{i}$, $C F_{i}=$ the corresponding simulated conflicts at the same site $i$, $L N=$ the natural logarithm, $L N(\alpha)=$ the model intercept, and $\beta=$ Regression coefficient

This is in lieu of the conventional expression relating crashes to traffic volume. To represent the before period (i.e. condition without treatment), a sample of untreated sites can be simulated to obtain the average number of conflicts at each site. Then, the parameters in Equation (5) can be obtained using the generalized linear models (GLM) technique, by relating the observed number of crashes and the simulated conflicts at each site, to represent the before period.

The calibrated parameters, from Equation (5), are then used to estimate the expected number of crashes at treated sites. Furthermore, to estimate the expected number of crashes in the after period $(\pi)$, at a certain site, the expected number of crashes in the before period (without treatment) is computed at the same site and then multiplied by a correction factor (Cr), similar to that for the conventional method. Usually the simulation conditions (e.g., traffic volumes, simulation parameters, driving behavior, etc.) will remain the same, except for the change due to the treatment, then this correction factor $(\mathrm{Cr})$ can be estimated based on the change in simulated conflicts in the before and after, as follows:

$C r=C_{a} / C_{b}$ 
where,

$\mathrm{Cr}=$ the correction factor
between the before and the after
periods;
$C_{a}=$ number of conflicts in the
after period (with treatment);
and
$C_{b}=$ number of conflicts in
the before period (without
treatment).

The correction factor "Cr" (in Eq. 6) can be viewed as a modification factor from the application of the simulation alone, based on the simulated conflicts in the before and the after (i.e. with and without treatment).

If we assume that volume and other factors remain constant in the before-and-after periods, we can expect that the only change in simulated conflicts and crashes take place as a result of treatment. The value of $\pi$ is obtained by summing over all sites in the treatment group $\left(\pi_{\text {sum }}\right)$ and compared with the observed number of crashes in the after period $\left(\lambda_{\text {sum }}\right)$. In addition, the variance of $\pi$ is also summed over all treated sites. The combined CMF at all sites is obtained by replacing $\lambda$ and $\pi$ in Equations (3) and (4) with their respective summations. For more details on the EB before-after analysis method for estimating a CMF, the reader is advised to read the Highway Safety Manual (AASHTO, 2010), and (Hauer, 1997).

\section{Data Description}

The data used in this study contains two samples of signalized intersections from Toronto, Canada. Sample 1 consists of 53 untreated reference intersections used to establish the SPF. Sample 2 consists of 47 treated intersections used to compare the conventional EB before-and-after treatment effect with the proposed crash-conflict formulation, as introduced in this paper. As noted above, the treatment implemented at the 47 sites reflects a change in left turn (LT) signal priority for a single approach from permissive to protected-permissive control.

The intersection traffic volumes and observed crashes are available from the Toronto Traffic Management Center. Observed crashes were available for the period from 1999 to 2007. The AM/PM peak hour traffic volumes and pedestrian counts for both the major and minor approaches were also available along with the intersections geometry. In addition, signal timing information, for all the untreated and treated intersections with and without LT signal priority treatment, were also available. Intersection geometry along with traffic volume and pedestrian counts were used as inputs into the simulation model.

\section{Simulation of Traffic Conflicts}

To use traffic conflicts in safety analysis, a surrogate safety indicators needs to be identified based on individual vehicle interactions. When vehicle interactions exceed a specified degree of risk, they are referred to as "traffic conflicts". Many surrogate safety measures (i.e. traffic conflict) expressions, such as Time-tocollision (TTC), Deceleration rate required to avoid crash (DRAC), etc. have been described in the literature (Gettman and Head, 2003; Archer, 2005, Cunto, 2008 and Cunto and Saccomanno, 2008). In this research study, Time-to-Collision (TTC) is used to reflect vehicle interactions. TTC can be expressed as the time required for two vehicles to crash if they continue on the same path and driving at their current 
speeds (Hayward, 1972).

The 53 untreated and the 47 treated signalized intersections were simulated using the VISSIM microscopic traffic simulation model, version 5.40, (PTV, 2012) to extract vehicles trajectories. Table 1 shows the VISSIM parameters used in the simulation of vehicles in this paper.

In our study, the AM and PM peak hours traffic volumes were simulated using VISSIM to extract vehicle trajectories for the treated and the untreated intersections. The obtained trajectories are then used to obtain traffic conflicts. Fifty VISSIM simulation runs with 5-minutes warmingup period (i.e. the Simulation Initialization Period) were used to simulate traffic at each intersection. It is worth noting that for each simulation run a unique random seed was used to capture the randomness in traffic at each intersection. To ensure that all vehicles entered the simulation network, a 2-hour simulation time was used.

The Surrogate Safety Assessment Model (SSAM) (Pu and Joshi, 2008) was used to obtain the traffic conflicts from the simulated vehicle trajectories. In this study the total number of traffic conflicts for two TTC thresholds: $\leq 1.50 \mathrm{~s}$, and $\leq 0.50 \mathrm{~s}$ were considered. The 1.50s TTC threshold reflects a lower level of risk that assumes vehicles are in potential conflicts if drivers have less than 1.50 s to perceive a danger and react accordingly. On the other hand, the $0.50 \mathrm{~s}$ TTC threshold reflects a much higher risk wherein a driver only have 0.50 s or less in which to take appropriate action to avoid a crash. These TTC thresholds have been used by other researchers (Shahdah et al., 2014; Cunto, 2008; Archer, 2005; etc.). The number of traffic conflicts at each site was calculated as an average from the 50 simulation runs.

Table 1

Simulation Behavioral Parameters

\begin{tabular}{|c|c|}
\hline Parameter & Value \\
\hline Driving Model & Urban (Motorized) \\
\hline Car-Following Model & Wiedemann 74 \\
\hline \multirow[t]{2}{*}{ Car-Following Parameters } & $\begin{array}{l}\mathrm{ax}=2.00 \mathrm{~m}^{*} \\
\mathrm{bx} \_ \text {add }=2.00^{* *} \\
\mathrm{bx} \text { _mult }=3.00^{* * *}\end{array}$ \\
\hline & Smooth close-up \\
\hline \multirow{3}{*}{ Conflict Areas ${ }^{* * * *}$} & Front gap $=0.00 \mathrm{~s}$ \\
\hline & Rear gap $=0.00 \mathrm{~s}$ \\
\hline & $\begin{array}{l}\text { Avoid Blocking }=0 \text { for all cases and }=1 \text { in case } \\
\text { of } 2 \text { left-turn lanes }\end{array}$ \\
\hline
\end{tabular}

${ }^{*}$ The average standstill distance with standard deviation of $0.30 \mathrm{~m}$;

${ }^{* *}$ The additive part of safety distance;

${ }^{* * *}$ The multiplicity part of safety distance;

${ }^{* * * *}$ Left turn only. 


\section{Calibration of Crash-Conflict SPF}

A total of 53 untreated signalized intersections were used to establish the empirical relationship between observed crashes and simulated conflicts. Table 2 summarizes traffic volume inputs used in the simulation for the treated and the untreated intersections. Table 3 summarizes observed rear-end (RE) and left-turn opposing (LTOPP) crashes at these intersections. Only vehicle-to-vehicle crashes have been considered in this study. LTOPP crashes refer to possible crashes between a leftturning vehicles and through vehicles in the opposing direction. Rear-end conflicts are considered to be affected by treatment since left-turn signal priority requires vehicles to slow down, especially if drivers are faced with a risky gap acceptance decision prior to initiating the left turn maneuver. This affects the likelihood of higher risk rear-end interactions with following vehicles in the traffic stream.

Table 4 summarizes simulation results for vehicle-to-vehicle conflicts with $\mathrm{TTC} \leq 1.50 \mathrm{~s}$ and $\mathrm{TTC} \leq 0.50 \mathrm{~s}$, respectively. It is worth noting that in our analysis we assume normal road, traffic and weather conditions, since the VISSIM parameters used in this study were calibrated for normal weather conditions. The observed crash data used in calibrating the crash-conflict SPF model were also filtered for normal weather conditions.

\section{Table 2}

Traffic Volume at Treated and Untreated Sites

\begin{tabular}{|c|c|c|c|c|c|c|}
\hline \multicolumn{7}{|c|}{ Untreated Sites } \\
\hline Statistic & $\begin{array}{c}\text { Volume in } \\
\text { Major }\end{array}$ & $\begin{array}{c}\text { Volume in } \\
\text { Minor }\end{array}$ & $\begin{array}{c}\text { \%RT } \\
\text { Major }\end{array}$ & \%RT Minor & \%LT Major & \%LT Minor \\
\hline Number & \multicolumn{6}{|c|}{53 sites } \\
\hline Average & 1301.62 & 764.91 & 10.63 & 16.07 & 6.36 & 12.04 \\
\hline SD $^{*}$ & 417.22 & 270.57 & 6.24 & 8.31 & 5.87 & 9.70 \\
\hline Maximum & 2246 & 1367 & 34 & 54 & 33 & 46 \\
\hline Minimum & 663 & 48 & 0 & 0 & 0 & 0 \\
\hline \multicolumn{7}{|c|}{ Treated Sites } \\
\hline $\begin{array}{c}\text { Summary } \\
\text { Statistic }\end{array}$ & $\begin{array}{c}\text { Volume in } \\
\text { Major }\end{array}$ & $\begin{array}{c}\text { Volume in } \\
\text { Minor }\end{array}$ & $\begin{array}{c}\text { \%RT } \\
\text { Major }\end{array}$ & \%RT Minor & \%LT Major & \%LT Minor \\
\hline Number & \multicolumn{6}{|c|}{47 sites } \\
\hline Average & 2549.43 & 1349.06 & 10.28 & 22.25 & 10.91 & 19.95 \\
\hline SD $^{*}$ & 934.08 & 761.98 & 7.15 & 16.37 & 5.01 & 13.01 \\
\hline Maximum & 4742 & 3340 & 40 & 81 & 26 & 66 \\
\hline Minimum & 847 & 157 & 2 & 5 & 1 & 0 \\
\hline
\end{tabular}

${ }^{*} S D$ : standard deviation

Table 3

Crash Data at Untreated Sites

\begin{tabular}{|c|c|c|}
\hline Statistic & Rear-End & LTOPP \\
\hline Sum & 915 & 309 \\
\hline Average & 17.26 & 5.83 \\
\hline SD & 11.34 & 5.56 \\
\hline Maximum & 48 & 20 \\
\hline Minimum & 2 & 0 \\
\hline
\end{tabular}


Table 4

Simulated Conflicts at Untreated Intersections

\begin{tabular}{|c|c|c|c|c|}
\hline \multirow{2}{*}{ Statistic } & \multicolumn{2}{|c|}{ TTC $\leq \mathbf{1 . 5 0 s}$} & \multicolumn{2}{c|}{ TTC $\leq \mathbf{0 . 5 0 s}$} \\
\cline { 2 - 5 } & Rear-End & LTOPP & Rear-End & LTOPP \\
\hline Sum & 348.00 & 29.78 & 17.16 & 8.62 \\
\hline Average & 6.57 & 0.56 & 0.32 & 0.16 \\
\hline SD & 5.58 & 0.44 & 0.37 & 0.17 \\
\hline Maximum & 23.98 & 1.82 & 1.98 & 0.90 \\
\hline Minimum & 0.36 & 0.00 & 0.00 & 0.00 \\
\hline
\end{tabular}

A Generalized Linear model (GLM) SPF functions, with assumed Negative Binomial (NB) error distribution, were fitted to the crash-conflict data for the 53 untreated sites, based on the SPF formula in Equation (5). The R-statistical software (R Development Core Team, 2019) was used to obtain the SPF model parameters. It is worth noting that the R-Software uses maximum likelihood method in estimating the GLM model parameters.

The SPFs estimates (with standard errors in parentheses) and goodness of fit for RE and LTOPP crashes are as follows:

1- RE crashes with TTC $\leq 1.50$ s:

$L N\left(C_{R E}\right)=2.07[0.15]+0.46[0.08] L N(C F)$

Dispersion Parameter $=0.195 ;$ ResidualDeviance $=53.85$

Degrees of freedom $=51 ; \quad 2 \log$ likelihood $=-364.52 ; \quad$ AIC $=370.52$

2- RE crashes with TTC $\leq 0.50$ s:

$L N\left(C_{R E}\right)=3.27[0.21]+0.216[0.096] L N(C F)$

Dispersion Parameter $=0.31$; ResidualDeviance $=55.18$

Degrees of freedom $=51 ; \quad 2 \log$ likelihood $=-385.58 ; \quad$ AIC $=391.58$

3- LTOPP crashes with $\mathrm{TTC} \leq 1.50 \mathrm{~s}$ :

$L N\left(C_{\text {LTOPP }}\right)=2.14[0.14]+0.58[0.13] L N(C F)$

Dispersion Parameter $=0.42$; ResidualDeviance $=59.51$

Degrees of freedom $=51 ; \quad 2 \log$ likelihood $=-279.55 ; \quad$ AIC $=285.55$

4- LTOPP crashes with TTC $\leq 0.50 \mathrm{~s}$ :

$L N\left(C_{L T O P P}\right)=2.80[0.30]+0.56[0.14] L N(C F)$

Dispersion Parameter $=0.53$; ResidualDeviance $=58.76$

Degrees of freedom $=51 ; \quad 2 \log$ likelihood $=-287.16 ; \quad$ AIC $=293.16$ 
All the parameters in Equations (7) - (10) are statistically significant at the $5 \%$ level. For LTOPP crashes with TTC $\leq 0.50 \mathrm{~s}$ and $\mathrm{TTC} \leq 1.50 \mathrm{~s}$, differences in the Akaike Information Criterion (AIC) were found to be less than 10. This suggests that there is no significant difference between the two LTOPP crash models with TTC $\leq 0.50 \mathrm{~s}$ and TTC $\leq 1.50 \mathrm{~s}$. On the other hand, the rear-end crash model with $\mathrm{TTC} \leq 1.50 \mathrm{~s}$ is better than the model that was obtained for TTC $\leq 0.50 \mathrm{~s}$, as the AIC value is significantly lower for TTC $\leq 1.50 \mathrm{~s}$.

(a)

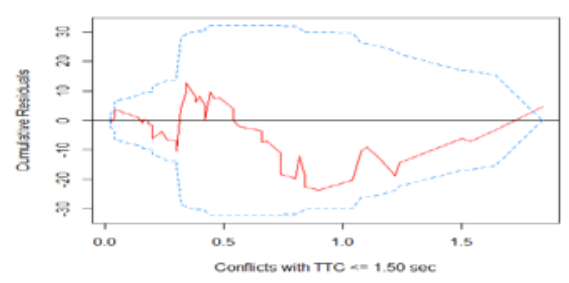

In addition to the AIC criterion, the Cumulative Residual (CURE) plots (Hauer and Bamfo, 1997) were obtained for the targeted crashes (i.e. LTOPP crashes) as shown in Fig. 1. for TTC $\leq 1.50$ s and $\mathrm{TTC} \leq 0.50 \mathrm{~s}$, respectively, and these plots suggest that the crash-conflict models provide a good fit for the observed crash data over the full range of conflict thresholds. It is worth noting that similar plots were obtained for RE crashes and conflicts with similar results.

(b)

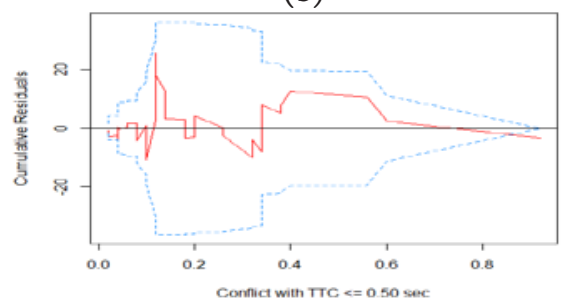

Fig. 1.

CURE Plots for LTOPP Conflicts with (a) TTC $\leq 1.50$ s and (b) TTC $\leq 0.50 \mathrm{~s}$

\section{Treated Sample}

Forty-seven (47) treated signalized intersections with only one treated approach, from Toronto, Canada, were used to evaluate the treatment effect. Traffic volume data at the treated intersections are summarized in Table 2. Table 5 provides a summary of the RE and LTOPP crash data used in this analysis.
It is worth noting that, the observed crashes show a reduction of $25 \%$ in $\mathrm{RE}$ crashes and $45 \%$ reduction in LTOPP crashes. These changes are due solely to the RTM bias and should not be used to guide treatment effect (LT phase change from permissive to protected permissive).

\section{Table 5}

Summary of Crashes for the Before and After at Treated Sites

\begin{tabular}{|c|c|c|c|c|}
\hline \multirow{2}{*}{ Statistic } & \multicolumn{2}{|c|}{ Rear End } & \multicolumn{2}{c|}{ LTOPP } \\
\cline { 2 - 5 } & Before & After & Before & After \\
\hline Sum & 1837 & 1383 & 558 & 314 \\
\hline Mean & 39.09 & 29.43 & 11.87 & 6.68 \\
\hline SD $^{*}$ & 35.10 & 22.98 & 9.94 & 6.13 \\
\hline Maximum & 125 & 88 & 36 & 32 \\
\hline Minimum & 0 & 0 & 0 & 0 \\
\hline
\end{tabular}

*SD: standard deviation 
Table 6 summarizes the traffic conflicts for $\mathrm{RE}$ and LTOPP conflicts for TTC $\leq 1.50 \mathrm{~s}$ and $\mathrm{TTC} \leq 0.50 \mathrm{~s}$, as obtained from the simulation of the 47 treated intersections.
It is worth noting that the simulation was carried out for the same traffic volumes with treatment and without treatment periods, for the corresponding signal priority phase.

Table 6

Simulated Conflicts for the Before and After at Treated Sites

\begin{tabular}{|c|c|c|c|c|}
\hline \multirow{2}{*}{ TTC Threshold } & Treatment Period & Statistic & Rear End & LTOPP \\
& \multirow{3}{*}{$\leq \mathbf{1 . 5 0 s}$} & Average & 1371.78 & 164.00 \\
& \multirow{2}{*}{ Before } & SD $^{*}$ & {$[34.85]$} & {$[11.77]$} \\
\cline { 2 - 5 } & \multirow{2}{*}{ After } & Average & 1296.42 & 118.70 \\
& & SD $^{*}$ & {$[34.77]$} & $10.13]$ \\
\hline \multirow{3}{*}{$\leq \mathbf{0 . 5 0 s}$} & \multirow{2}{*}{ Before } & Average & 12.28 & {$[4.49]$} \\
\cline { 2 - 5 } & \multirow{2}{*}{ After } & SD $^{*}$ & {$[3.59]$} & 11.72 \\
& & Average & {$[3.68]$} & {$[4.19]$} \\
\hline
\end{tabular}

"SD: standard deviation

\section{Crash Modification Factors (CMFs) for treated intersections}

Table 7 summarizes the results of the application of crash-conflict EB before-andafter analysis for the treated intersection sample. These results consider rear-end and
LTOPP crashes and two TTC thresholds. Increasing TTC threshold from 1.50 s to $0.50 \mathrm{~s}$ has the effect of reducing the effectiveness of the treatment for LTOPP crashes, and this is expected since the lower risk threshold (TTC $\leq 1.5 \mathrm{~s}$ ) is expected to pick up a larger number of conflicts in the simulation.

Table 7

CMFs at Treated Intersections Using Proposed Method

\begin{tabular}{|c|c|c|c|c|c|c|}
\hline \multirow{2}{*}{ Crash Type } & $\begin{array}{c}\text { TTC } \\
\text { Threshold }\end{array}$ & $\lambda_{\text {sum }}{ }^{*}$ & $\pi_{\text {sum }}^{* *}$ & VAR( $\left.\boldsymbol{\pi}_{\text {sum }}\right)$ & CMF & SE \\
\hline \multirow{2}{*}{ Rear-end } & $\leq 1.50 \mathrm{~s}$ & 1383 & 1361.650 & 1822.852 & $1.015^{* * *}$ & 0.042 \\
\cline { 2 - 7 } & $\leq 0.50 \mathrm{~s}$ & 1383 & 1287.659 & 1647.704 & $1.073^{* * * *}$ & 0.044 \\
\hline \multirow{2}{*}{ LTOPP } & $\leq 1.50 \mathrm{~s}$ & 314 & 454.406 & 587.015 & $0.689^{* * *}$ & 0.053 \\
\cline { 2 - 7 } & $\leq 0.50 \mathrm{~s}$ & 314 & 448.799 & 581.974 & $0.698^{* * *}$ & 0.054 \\
\hline
\end{tabular}

"Sum of the observed crashes in the after period;

${ }^{* *}$ Sum of the expected crashes in the after period;

${ }^{* *}$ Statistically significantly different from EB estimate at $5 \%$ confidence level;

${ }^{* * * *}$ Not statistically significantly different from EB estimate at $5 \%$ confidence level.

The CMF estimates are compared with values from an EB before-and-after analysis that uses the conventional prior (crashes verses traffic volumes and road geometric attributes) in the EB formulation, for the same treatment and exactly the same treated 
intersections from Toronto, Canada. The results of the conventional EB before-andafter study by Srinivasan et al. (2011 and 2012), for the same sites and treatment, are summarized in Table 8 . It is worth noting that a reference group of 626 untreated signalized intersections were used by Srinivasan et al. (2011 and 2012) in their conventional EB model to calibrate the SPF (the prior in the EB structure) as a function of traffic volumes, number of approaches, presence of LT lanes, etc.

\section{Table 8}

Conventional EB Before-and-After CMFs

\begin{tabular}{|c|c|c|c|c|}
\hline Crash Type & $\lambda_{\text {sum }}{ }^{*}$ & $\pi_{\text {sum }}{ }^{* *}$ & CMF & SE \\
\hline Rear-end & 1266 & 1383 & 1.091 & 0.046 \\
\hline LTOPP & 341 & 314 & 0.919 & 0.069 \\
\hline
\end{tabular}

"Sum of the observed crashes in the after period;

${ }^{* *}$ Sum of the expected crashes in the after period.

(Source: Srinivasan et al., 2012)

The CMF estimates (in Table 7 and Table 8) for rear-end crashes with $\mathrm{TTC} \leq 0.50 \mathrm{~s}$ are comparable to the CMF values from the conventional EB before-and-after analysis, as there is no statistically significant different between them at the 5\% level. However, as the threshold is increased the difference becomes significant at the $5 \%$ level. For LTOPP crashes, CMF estimates were found to be significantly different at the $5 \%$ level for both TTC thresholds. The result suggest that the crash-conflict model shows treatment to be more effective in reducing LTOPP crashes than was predicted by the conventional EB model. This is intuitively more logical since we expect LTOPP conflicts to be significantly reduced by this type of treatment. It is worth noting that the standard error (SE) associated with CMF estimates from the proposed method is smaller than those from the conventional EB for both RE and LTOPP crashes and for both TTC thresholds.

It is worth noting that the same intersections and treatment were used in other traffic conflict analyses, by Shahdah et al. (2014 and 2015), to estimate CMFs based mainly on simulated traffic conflicts. The results in this research paper is consistent with the results from Shahdah et al. (2014 and 2015).

\section{Conclusions}

This study suggests that simulated traffic conflicts can be used instead of traffic volumes to enhance the performance of EB before-and-after analysis for estimating treatment effects. The integration of traffic conflicts in the EB model requires the development of a crash-conflict relationship (i.e., to estimate priors) and the simulation of the treated sites with and without treatment for the same traffic volume to reflect treatment effects only.

A treated signalized intersection sample from Toronto was used to obtain CMF estimates from the proposed method and to compare results with conventional EB estimates. CMF estimates for rear-end crashes at high risk TTC threshold (i.e., $\mathrm{TTC} \leq 0.50 \mathrm{~s}$ ) are similar to estimates from conventional EB. On the other hand, CMF estimates for LTOPP suggests a more 
significant reduction in crashes at treated sites than was obtained from conventional EB before and after analysis. These results are more intuitive since we would expect the reduction of LTOPP conflicts to be the primary focus of the prohibited LT treatment. Furthermore, the standard error associated with CMF estimates from the proposed method is lower than those obtained from conventional EB for both rear-end and LTOPP crashes and both TTC thresholds. As the proposed method is based mainly on simulated conflicts, it has the added advantage to account for high-risk vehicles interactions that are by default not considered in the reported crashes by police.

\section{Acknowledgments}

The Toronto Traffic Management Center (the Traffic Safety Unit) is gratefully acknowledged for providing the data used in this research.

\section{References}

AASHTO. 2010. Highway safety manual, 1st edition. American Association of State Highway and Transportation Officials, Washington, D.C.

Allen, B. L.; Shin, B. T.; Cooper, P. J. 1978. Analysis of Traffic Conflicts and Collisions, Transportation Research Record: Journal of the Transportation Research Board 667: 67-74.

Amundsen, F.; Hyden, C. 1977. The Swedish traffic conflict technique. In Proceedings of First Workshop on Traffic Conflicts, Institute of Transport Economics. Oslo, Norway, 1-5.

Archer, J. 2005. Indicators for traffic safety assessment and prediction and their application in micro-simulation modelling: A study of urban and suburban intersections. $\mathrm{PhD}$ thesis, Royal Institute of Technology, Kungliga Tekniska Högskolan (KTH), SE-100 44 Stockholm, Sweden.
Brown, G.R. 1994. Traffic conflicts for road user safety studies, Canadian Journal of Civil Engineering 21: 1-15.

Cunto, F. 2008. Assessing Safety Performance of Transportation Systems using Microscopic Simulation. PhD dissertation, University of Waterloo, Ontario, Canada.

Cunto, F.; Saccomanno, F.F. 2008. Calibration and validation of simulated vehicle safety performance at signalized intersections, Accident Analysis and Prevention 40: 1171-1179.

Gettman, D.; Head, L. 2003. Surrogate safety measures from traffic simulation models, Transportation Research Record: Journal of the Transportation Research Board 1840: 104-115.

Hauer, E.; Bamfo, J. 1997. Two Tools for Finding What Function Links the Dependent Variable to the Explanatory Variables. In Proceedings of International Cooperation on Theories and Concepts in Traffic Safety (ICTCT). Lund, Sweden, 1-7.

Hauer, E. 1997. Observational Before-After Studies in Road Safety: Estimating the Effect of Highway and Traffic Engineering Measures on Road Safety. Pergamon Press, Elsevier Science Ltd., Oxford, U.K. 306 p.

Hayward, J. C. 1972. Near-miss Determination through Use of a Scale of Danger, Highway Research Record 384: 24-34.

Lan, B. 2010. Exploration of Theoretical and Application Issues in Using Fully Bayes Methods for Road Safety Analysis. $\mathrm{PhD}$ dissertation, Ryerson University, Ontario, Canada.

Lopez, P.A.; Behrisch, M.; Bieker-Walz, L.; Erdmann, J.; Flötteröd, Y.P.; Hilbrich, R.; Lücken, L.; Rummel, J.; Wagner, P.; WieBner, E. 2018. Microscopic traffic simulation using sumo. In $201821^{\text {st }}$ International Conference on Intelligent Transportation Systems (ITSC), 2018 Nov 4, 2575-2582. IEEE. [Computer Software]

Persaud, B.; Lyon, C. 2007. Empirical Bayes before-after safety studies: Lessons learned from two decades of experience and future directions, Accident Analysis and Prevention 39(3): 546-555. 
PTV. 2012. Planung Transport Verkehr, VISSIM 5.40 User Manual. AG, Stumpfstraße 1, D-76131 Karlsruhe, Germany. [Computer Software].

Pu, L.; Joshi, R. 2008. Surrogate Safety Assessment Model (SSAM): Software User Manual. Publication FHWA-HRT-08-050. Federal Highway Administration, U.S. Department of Transportation. 100 p. Available from internet: <http://www.fhwa.dot.gov/publications/ research/safety/08050/08050.pdf $>$.

Quadstone Paramics Ltd. 2019. Computer Software. Available from internet: $<$ http://www.paramics-online. com $>$.

R Development Core Team. 2019. R: A Language and Environment for Statistical Computing. R 13 Foundation for Statistical Computing, Vienna, Austria. [Computer Software]. Available from internet: <http://www.R-14 project.org>.

Sayed, T.; Brown, G; Navin, F. 1994. Simulation of traffic conflicts at unsignalized intersections with TSC-sim, Accident Analysis and Prevention 26: 593-607.

Shahdah, U.; Saccomanno, F.; Persaud, B. 2014. Integrated traffic conflict model for estimating crash modification factors, Accident Analysis and Prevention 71: 228-235.

Shahdah, U.; Saccomanno, F.; Persaud, B. 2015. Application of traffic microsimulation for evaluating safety performance of urban signalized intersections, Transportation Research Part C: Emerging Technologies 60: 96-104.
Shahdah, U. 2014. Integrating Observational and Microscopic Simulation Models for Traffic Safety Analysis. PhD thesis, University of Waterloo, Canada.

Srinivasan, R.; Gross, F.; Lyon, C.; Persaud, B.; Eccles, K.; Hamidi, A.; Baek, J.; Smith, S.; Lefler, N.; Sundstrom, C.; Carter, D. 2011. Evaluation of Safety Strategies at Signalized Intersections. NCHRP Report 705 .

Srinivasan, R.; Lyon, C.; Persaud, B.; Baek, J.; Gross, F.; Smith, S.; Sundstrom, C. 2012. Crash modification factors for changes to left-turn phasing, Transportation research record 2279(1): 108-117.

Tsoi, K. 2004. Inventory of Urban Health Related Data covering the Greater Toronto Area-Supporting Documentation: Report on the Availability of Inter- and Intra-city Traffic Volume Data for Toronto, Windsor and Montreal. Available from internet: <http://www.cuhi. utoronto.ca/data_inventory/supportingdocuments/ inter_intratraffic.html>.

TSS. 2019. Transport Simulation Systems, Computer Software. Available from internet: <http://www.aimsun. com $>$.

Van der Horst, A.R.A. 1990. A Time-based Analysis of Road User Behaviour in Normal and Critical Encounters. $\mathrm{PhD}$ thesis, Delft University of Technology. 